\title{
Testing the Relationship between Bursty Star Formation and Size Fluctuations of Local Dwarf Galaxies
}

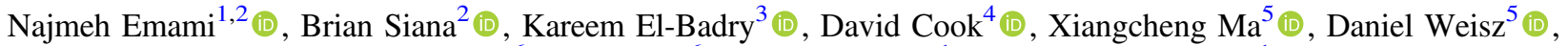 \\ Joobin Gharibshah $^{6}$, Sara Alaee ${ }^{6}$, Claudia Scarlata ${ }^{1}$ (10), and Evan Skillman ${ }^{1}$ (1) \\ ${ }^{1}$ Minnesota Institute for Astrophysics, University of Minnesota, 116 Church St. SE, Minneapolis, MN 55455, USA; nemami@umn.edu \\ ${ }^{2}$ Department of Physics and Astronomy, University of California Riverside, Riverside, CA 92521, USA \\ ${ }^{3}$ Center for Astrophysics, Harvard \& Smithsonian, 60 Garden Street, Cambridge, MA 02138, USA \\ ${ }^{4}$ Infrared Processing and Analysis Center, California Institute of Technology, Pasadena, CA, USA \\ ${ }^{5}$ Department of Astronomy and Theoretical Astrophysics Center, University of California Berkeley, Berkeley, CA 94720, USA \\ ${ }^{6}$ Computer Science and Engineering Department, University of California, Riverside, CA 92521, USA \\ Received 2021 June 4; revised 2021 August 12; accepted 2021 August 18; published 2021 December 1
}

\begin{abstract}
Stellar feedback in dwarf galaxies plays a critical role in regulating star formation via galaxy-scale winds. Recent hydrodynamical zoom-in simulations of dwarf galaxies predict that the periodic outward flow of gas can change the gravitational potential sufficiently to cause radial migration of stars. To test the effect of bursty star formation on stellar migration, we examine star formation observables and sizes of 86 local dwarf galaxies. We find a correlation between the $R$-band half-light radius $\left(R_{e}\right)$ and far-UV luminosity $\left(L_{\mathrm{FUV}}\right)$ for stellar masses below $10^{8}$ $M_{\odot}$ and a weak correlation between the $R_{e}$ and $\mathrm{H} \alpha$ luminosity $\left(L_{\mathrm{H} \alpha}\right)$. We produce mock observations of eight lowmass galaxies from the FIRE-2 cosmological simulations and measure the similarity of the time sequences of $R_{e}$ and a number of star formation indicators with different timescales. Major episodes of $R_{e}$ time sequence align very well with the major episodes of star formation, with a delay of $\sim 50 \mathrm{Myr}$. This correlation decreases toward star formation rate indicators of shorter timescales such that $R_{e}$ is weakly correlated with $L_{\mathrm{FUV}}(10-100 \mathrm{Myr}$ timescale) and is completely uncorrelated with $L_{\mathrm{H} \alpha}$ (a few Myr timescale), in agreement with the observations. Our findings based on FIRE-2 suggest that the $R$-band size of a galaxy reacts to star formation variations on a $\sim 50 \mathrm{Myr}$ timescale. With the advent of a new generation of large space telescopes (e.g., JWST), this effect can be examined explicitly in galaxies at higher redshifts, where bursty star formation is more prominent.
\end{abstract}

Unified Astronomy Thesaurus concepts: Dwarf galaxies (416); Galaxy kinematics (602); Galaxy dynamics (591); Star formation (1569); Galaxy evolution (594)

Supporting material: figure set

\section{Introduction}

Flat cosmological models with a mixture of dark energy, cold dark matter, and baryonic matter are consistent with the observations of structure formation on large scales ( $\gg 1 \mathrm{Mpc}$; Bahcall et al. 1999; Frenk \& White 2012; Vogelsberger et al. 2014; Schaye et al. 2015; Planck Collaboration et al. 2020). In this cold dark matter model $(\Lambda \mathrm{CDM})$, dark matter is viewed as particles with weak self-interactions, as well as negligible interactions with baryonic matter. However, on galactic scales $(<1 \mathrm{Mpc})$ there are several tensions between observations and predictions of $\Lambda \mathrm{CDM}$ (for a comprehensive review see Bullock \& Boylan-Kolchin 2017).

Dwarf galaxies, with stellar masses $\lesssim 10^{9} M_{\odot}$, are known to challenge CDM predictions on small scales (Brooks 2019). It has long been recognized that the observed dark matter halos of dwarf galaxies exhibit a different core density profile (Flores \& Primack 1994; Moore 1994) than the predicted dark-matter-only Navarro-Frenk-White (NFW; Navarro et al. 1997) profile.

The NFW density profile rises steeply at small radii (Navarro et al. 2010). However, observations of rotation curves of dwarf galaxies reveal rather a constant-density profile in apparent contrast with the NFW profile (McGaugh et al. 2001; Marchesini et al. 2002; Simon et al. 2005; Kuzio de Naray et al. 2008; Oh et al. 2015; Relatores et al. 2019), referred to as the "cusp-core" problem. Two solutions have been proposed in the literature.
The first solution is a modification to the $\Lambda \mathrm{CDM}$ in which dark matter particles are posited to be strongly self-interacting (Spergel \& Steinhardt 2000; Kaplinghat et al. 2016). In the selfinteracting dark matter model, dark matter collisions allow energy exchange between particles and thermalize the inner halo over the cosmological timescale, leading to a cored central density (Vogelsberger et al. 2012; Peter et al. 2013; Elbert et al. 2015; Fry et al. 2015; Sameie et al. 2020).

The second solution to the cusp-core problem is to implement baryonic feedback (in forms of supernova explosions, radiation pressure, photoelectric heating, photoionization, and stellar winds; Navarro et al. 1996; Governato et al. 2010; Sales et al. 2014; Hopkins et al. 2020). In these models, outflows that are driven by baryonic feedback over the course of star formation (SF) are capable of inducing fluctuations in the central gravitational potential of the galaxy, transferring energy into dark matter particles, and ultimately flattening the dark matter core density profile in dwarf galaxies (Governato et al. 2010; Sales et al. 2010; Pontzen \& Governato 2012; Chan et al. 2015; Read et al. 2016; Tollet et al. 2016). This alternative solution requires highresolution hydrodynamical simulations to model the inhomogeneous interstellar medium and couple the SF to high-density gas regions (Hopkins et al. 2014, 2018). Implementation of baryonic feedback into CDM-only simulations has been shown to further explain several other characteristics of dwarf galaxies such as their low stellar-to-halo mass ratio (Hirschmann et al. 2012; Behroozi et al. 2013; Moster et al. 2013). 
Other than forming a cored dark matter density profile, baryonic feedback is predicted to have additional impacts on the dynamical and morphological properties of dwarf galaxies (El-Badry et al. 2016, 2017; Read et al. 2019), many of which are shown to be in agreement with recent observations. For instance, outflows caused by supernova explosions result in bursty SF rates (SFRs) in dwarf galaxies (McQuinn et al. 2010; Sparre et al. 2017), which is consistent with the different SFRs inferred from SFR indicators that probe different timescales in local and high-redshift dwarf galaxies (Weisz et al. 2012; Domínguez et al. 2015; Guo et al. 2016; Mehta et al. 2017; Caplar \& Tacchella 2019; Emami et al. 2019; Faisst et al. 2019; Flores Velázquez et al. 2021). Outflows can also explain the lack of coherent disk formation in most irregular dwarf galaxies (Wheeler et al. 2017), as well as an observed specific SFR-gas velocity dispersion (sSFR- $\sigma$ ) correlation in this mass range (Stilp et al. 2013; Cicone et al. 2016; Hirtenstein et al. 2019; Pelliccia et al. 2020). Furthermore, outflowing winds that are powered by episodic bursts of SF drive radial migration of star and gas particles over the course of SF (De Young \& Heckman 1994; Graus et al. 2019; Mercado et al. 2021), which is consistent with metallicity and inverted age gradients in lowmass galaxies (Aparicio \& Tikhonov 2000; Stinson et al. 2009; Hidalgo et al. 2013; Vargas et al. 2014; McQuinn et al. 2017; Jafariyazani et al. 2019).

The predicted radial migration has another consequence: the outflowing winds can also impact the size of the galaxy. In particular, during bursts of SF gas and stars move into the center of the galaxy until the first supernova explodes. Subsequent supernova explosions will yield a shallow gravitational potential, gas and stellar migration toward larger radii resulting in more extended galaxy morphologies. Over time, the expelled gas will cool and collapse back onto the galaxy center, triggering the next burst of SF. This causes size fluctuations during bursts of SF across the galaxy (El-Badry et al. 2016). Yet this prediction has not been observationally confirmed for the real dwarf galaxies.

In this work, we aim to test this prediction for a sample of local dwarf galaxies with a stellar mass range of $10^{7}-10^{9.6} M_{\odot}$, the same regime suggested by El-Badry et al. (2016). We will study the relationship between bursty SF and size fluctuations in both observed and simulated galaxies and provide physical intuitions for our findings.

An overview of this paper is as follows. We describe the observed sample and our measurements of the galaxies' stellar mass, dust-corrected luminosities, and $R$-band size in Section 2. In Section 3 we discuss our observed findings. In Section 4 we analyze a suite of simulated dwarf galaxies, analogous to our observed sample, from FIRE-2 simulations and provide physical interpretations about the effect of bursty SF on the galaxy's size fluctuation. We discuss previous works and the implications for the era of the James Webb Space Telescope in Section 5. Lastly, we summarize our results in Section 6.

Throughout this work we assume that the default $\Lambda \mathrm{CDM}$ cosmology has parameters $H_{0}=67.4 \mathrm{~km} \mathrm{~s}^{-1} \mathrm{Mpc}^{-1}, \Omega_{m}=0.315$, and $\Omega_{\Lambda}=0.685$ (Planck Collaboration et al. 2020).

\section{Observations and Measurements}

In this work, we use a sample of galaxies from the Local Volume Legacy (LVL) survey (Kennicutt et al. 2008). LVL is an unbiased and statistically complete sample of 258 nearby field star-forming galaxies. Galaxies in the LVL sample are selected based on a set of criteria including galaxies that are within $11 \mathrm{Mpc}(D \leqslant 11)$, lie outside the Galactic plane (| $b \mid>20^{\circ}$ ), are brighter than $B=15 \mathrm{mag}$, and span an RC3 type galaxy range $T \geqslant 0$ (i.e., galaxies of spiral and irregular morphologies later than S0a). Due to its volume-limited nature, the LVL sample is dominated by dwarf galaxies and is complete above $10^{6.5} M_{\odot}$ stellar mass. The LVL public data set consists of GALEX ultraviolet (Lee et al. 2011), narrowband $\mathrm{H} \alpha$ imaging (Kennicutt et al. 2008), Spitzer IRAC and MIPS imaging (Dale et al. 2009), optical MMT spectroscopy (Berg et al. 2012), and optical ground-based imaging (Cook et al. 2014).

About $70 \%$ of the sample has deeper $R$-band imaging $(\sim 0.5$ mag deeper than the Sloan Digital Sky Survey (SDSS); Cook et al. 2014) obtained from 1-2 m telescopes located at Cerro Tololo Inter-American Observatory, Kitt Peak National Observatory, Vatican Advanced Technology Telescope, and the Wyoming Infra-Red Observatory. The median pixel scale of the instruments is 0 " 5 pixel $^{-1}$, with a standard deviation of 0 .' 1 pixel $^{-1}$. For the other $30 \%$ of the sample, we use SDSS $r$ band imaging taken from SDSS DR7 (Abazajian et al. 2009). We use the data product produced in Cook et al. (2014). All the images are sky subtracted and corrected for the contaminant sources, e.g., background galaxies and foreground stars.

The $\mathrm{H} \alpha$ flux measurement is described extensively in Kennicutt et al. (2008). Different aperture configuration methods were applied on galaxies in the sample to ensure that the $\mathrm{H} \alpha$ flux is integrated over the entire galaxy, not a subset.

The stellar masses and dust extinctions are obtained from Weisz et al. (2012) and Johnson et al. (2013). Briefly, the masses are derived by fitting the observed UV, optical, and IR luminosities of each galaxy with the Bruzual \& Charlot (2003) suite of stellar population synthesis models and a varying set of parameters (age, stellar metallicity, exponentially declining SF histories (SFHs), and dust). The $\mathrm{H} \alpha$ and UV dust extinctions are derived from the "energy balance" extinction correction method (Kennicutt et al. 2009; Hao et al. 2011), by which the intrinsic luminosity is retrieved from the combination of unobscured (e.g., $\mathrm{H} \alpha$ and UV) and obscured (far-IR continuum) signatures of SF. For the $30 \%$ of the sample where farIR measurements are not available, a correlation-based attenuation correction is used (Lee et al. 2009). For that, the $\mathrm{H} \alpha$ dust attenuation is determined via a scaling relation between $A_{\mathrm{H} \alpha}$ and $B$-band magnitude ( $M_{B}$; Lee et al. 2009). As shown in Lee et al. (2009), the scatter between $A_{\mathrm{H} \alpha}$ and $M_{B}$ decreases with decreasing luminosity, such that for the relatively transparent dwarf galaxies that dominate our sample $\left(-18<M_{B}<-14.7\right)$ the scatter is roughly $10 \%-20 \%$. The $\mathrm{H} \alpha$ extinction corrections used here yield similar results to those used by Lee et al. (2009). The dust corrections derived by the application of energy balance and the empirical $A_{\mathrm{H} \alpha}-M_{B}$ correlation are much smaller than the observed scatter in the $\mathrm{H} \alpha$ luminosity and do not have a significant effect on our interpretations about the bursty SF of the sample. The UV dust attenuation $\left(A_{\mathrm{UV}}\right)$ is also estimated as the $\mathrm{H} \alpha$ attenuation $\left(A_{\mathrm{H} \alpha}\right)$ scaled by 1.8 .

We determine the sizes of the galaxies in the $R$-band images. This is because the galactic potential fluctuations caused by the outflowing winds impact the distribution of all stars of any age. Additionally, through the $R$-band imaging, light from both young and old stellar populations can be assessed via the combination of strong nebular lines (i.e., $\mathrm{H} \alpha$, which traces stars 
that are formed a few Myr ago) and the stellar optical continuum (at a wavelength range of 5800-7300 $\mathrm{\text { }}$, which probes stars that are formed over hundreds of Myr). We run Source Extractor (Bertin \& Arnouts 1996) to determine the half-light radii in the $R$ band. Since the images are sky subtracted, we set the background value to zero in the code. For each galaxy, the zero-point, pixel scale, and gain parameters are set by values stored in the header files. These parameters depend on the characteristics of the telescope with which the object was observed. We set the FWHM parameter to the seeing of the observation. We choose a flexible elliptical aperture to measure the flux of the detected objects inside that aperture as described in Kron (1980). Whenever the image is highly resolved, which is mostly the case for objects that are closer than $3 \mathrm{Mpc}$, the Source Extractor algorithm segments the galaxy into multiple sources. In these cases we smooth the image by applying a Gaussian kernel with a minimum FWHM necessary for Source Extractor to recognize the galaxy as a single source. It may be of concern that the smoothing procedure washes out the galaxies' substructures, such as the spiral arms; therefore, the measured half-light radii are smaller than when the smoothing is not applied. However, the convolution that we perform is with a much (factor of $\sim 5$ ) smaller profile than the ultimate sizes measured in these galaxies. This means that the measured sizes will not be significantly increased by performing this convolution. Source Extractor yields the half-light radius from a circular photometric aperture within which 50\% of the total flux of the galaxy is enclosed. The choice of circular aperture is an acceptable approximation since the galaxies are mostly circular or irregular (few edge-on disks). For a sanity check, we also run GALFIT (Peng et al. 2002) for galaxies in the sample. We fit our galaxies with the Sersic profiles and a free Sersic index. Giving the Source Extractor parameters as an initial guess to GALFIT, we get half-light radii that are consistent with those from Source Extractor. We compare the half-light radii derived from Source Extractor and GALFIT for a subsample of our galaxies in Figure 1. The errors derived from the GALFIT algorithm are very small, with a median value of $2 \%$.

To be consistent with El-Badry et al. (2016), we only keep galaxies with axis ratios $>0.5$ and stellar masses between $10^{7}$ and $10^{9.6} M_{\odot}$, where burstiness is predicted to be more prominent compared to other mass ranges (El-Badry et al. 2016). This leaves us with a final sample of 86 galaxies.

\section{Results}

We present our size measurements as a function of stellar mass in Figure 2. The logarithm of half-light radius $\left(R_{e}\right)$ in our LVL sample linearly increases with the logarithm of mass with a slope of $0.282 \pm 0.025$. Our $R_{e}$ values are in agreement with the $r$-band effective radii of SDSS $\mathrm{z} \sim 0$ galaxies measured in the NASA-Sloan Atlas (Blanton et al. 2011).

At fixed mass, there is $\sim 0.8$ dex scatter inferred by the standard deviation. This scatter is the main driver of this work and is predicted to be partially driven by the feedback-driven outflows generated by episodic bursts of SF (El-Badry et al. 2016). Here we aim to examine this prediction and see whether there is any relationship between the size fluctuation and SF change in the observed low-mass galaxies.

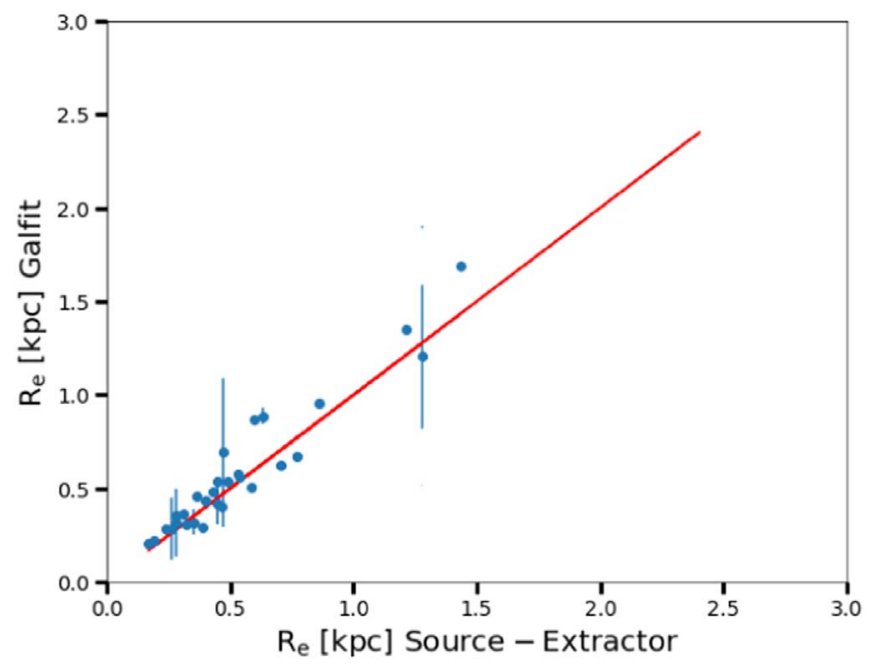

Figure 1. A comparison between the half-light radii $\left(R_{e}\right)$ derived from Source Extractor and GALFIT algorithms for a subsample of galaxies. The errors from GALFIT are very small, with a median value of $2 \%$.

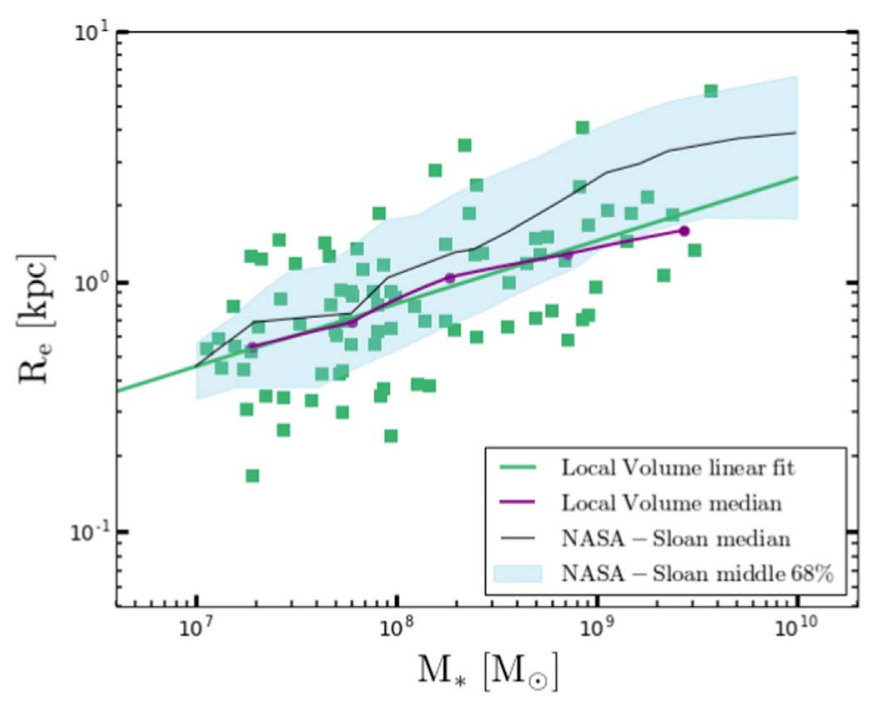

Figure 2. $R$-band half-light radius $\left(R_{e}\right)$ as a function of stellar mass for the LVL sample (green points). There is a correlation between the two quantities in the $\log$ scale with a slope of $0.282 \pm 0.025$ shown as a green line. The purple points are the median of the LVL sample for five mass bins. The typical $R_{e}$ errors for the individual galaxies are about $2 \%$. The blue shaded area and the black curve are the median and $1 \sigma$ scatter, respectively, of the NASA-Sloan Atlas at $z \sim 0$ (Blanton et al. 2011). The median of the NASA-Sloan sample is very close to the median and the linear fit to our LVL sample. The standard deviation is $\sim 0.8$ dex in $R_{e}$ at $10^{8} M_{\odot}$ stellar mass.

\subsection{Observed Size-Luminosity Relation}

In order to test the relation between the size and the SFR (or, equivalently, the luminosity) of the sample, we need to first subtract off the mass dependency from the $R_{e}$ distribution. In particular, at any given mass, we are interested in each galaxy's deviation in $R_{e}$ from the average trend shown in Figure 2. For this, we introduce a new definition of size, which is the ratio of $R_{e}$ to the average $R_{e}\left(R_{e} / \overline{R_{e}}\right)$. Technically, at each point we divide the observed $R_{e}$ by the average $\overline{R_{e}}$ derived from the line fit through the $\log \left(R_{e}\right)-\log \left(M_{*}\right)$ relation and refer to it as normalized-to-average size.

Figure 3 shows postage stamps of $R$-band images of a sample of LVL galaxies that are scaled by their distances as if they are all at the same distance and then are sorted by their 


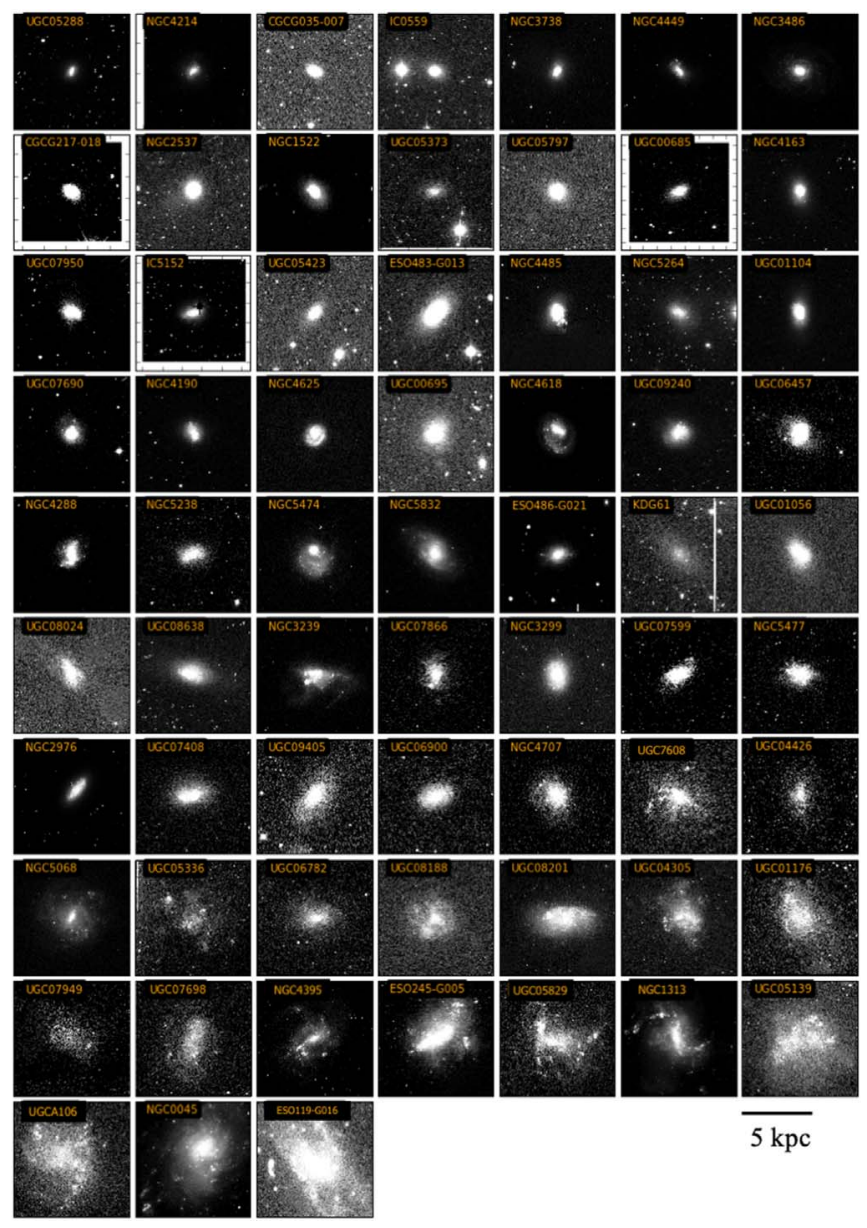

Figure 3. Postage stamps of $R$-band images of the LVL sample that are scaled to follow the fit relation shown in Figure 2 and are also scaled by their distance and are sorted by their $R_{e} / \overline{R_{e}}$ (half-light radius relative to the mean). Each stamp is labeled by the galaxies' LVL ID name.

$R_{e} / \overline{R_{e}}$. The top left panel shows the most compact galaxy in the sample with the smallest $R_{e} / \overline{R_{e}}$, while the bottom right panel indicates the galaxy with the largest $R_{e} / \overline{R_{e}}$.

Next, we calculate the dust-corrected $\mathrm{H} \alpha$ and far-UV (FUV) luminosities of the sample (Weisz et al. 2012). $\mathrm{H} \alpha$ and FUV luminosities are the two common probes of the SFRs in galaxies with timescales of 5 and 10-100 Myr respectively, depending on the SF systems (Kennicutt 1998; Emami et al. 2019; Flores Velázquez et al. 2021).

In order to take out the mass dependency from the $\mathrm{H} \alpha$ (and FUV) luminosity distributions, we divide the $\mathrm{H} \alpha$ (FUV) luminosity at a given mass by the average luminosity derived from the line fit through the $\log L_{\mathrm{H} \alpha}\left(\log L_{\mathrm{UV}}\right)$ and $\log M_{*}$ relation at that mass, the same procedure we used to derive the normalized-to-average size values $\left(R_{e} / \overline{R_{e}}\right)$. We then split the sample into four mass bins with equal number of galaxies in each bin and investigate the size-luminosity relation in each mass bin assuming that galaxies of similar stellar masses likely share common SF properties (Weisz et al. 2012; Emami et al. 2019). In particular, lower-mass galaxies should have a lower potential well and thus may be more likely to show signs of episodic SF in their sizes.

Figure 4 shows the logarithm of normalized-to-average $\mathrm{H} \alpha$ and FUV luminosities, namely, $L_{\mathrm{H} \alpha} / \overline{L_{\mathrm{H} \alpha}}$ and $L_{\mathrm{FUV}} / \overline{L_{\mathrm{FUV}}}$ as a function of $\log \left(R_{e} / \overline{R_{e}}\right)$ for four mass bins. We find a positive correlation between $\log \left(L_{\mathrm{FUV}} / \overline{L_{\mathrm{FUV}}}\right)$ and $\log \left(R_{e} / \overline{R_{e}}\right)$ at masses $<10^{7.85} M_{\odot}$. The correlation becomes weaker and more scattered toward larger mass bins. For the $\log \left(L_{\mathrm{H} \alpha} / \overline{L_{\mathrm{H} \alpha}}\right)-$ $\log \left(R_{e} / \overline{R_{e}}\right)$ relation, we also see a positive slope but less steep and more scattered than the $\log \left(L_{\mathrm{FUV}} / \overline{L_{\mathrm{FUV}}}\right)-\log \left(R_{e} / \overline{R_{e}}\right)$ relation. We report the slopes of the best-fit relationships for each mass bin and the Spearman rank correlation coefficients in Table 1. A larger Spearman rank correlation coefficient indicates a stronger correlation between two quantities.

In order to better understand these observed trends, we compare to dwarf galaxies in hydrodynamical zoom-in simulations.

\section{Hydrodynamical Simulations}

In Section 3, we found that for masses below $10^{7.85} M_{\odot}$ the normalized-to-average size is correlated with the FUV normalized-to-average luminosity at fixed stellar mass. This supports predictions from hydrodynamical simulations (El-Badry et al. 2016) suggesting that the size fluctuation and stellar migration in low-mass systems are consequences of feedback-driven outflows, which in turn are driven by the cumulative effects of many starburst episodes over time.

Based on this argument, SF is triggered when cold gas accretes into the center of the galaxy and forms stars. Stellar feedback then creates a galactic wind and drives gas to larger radii, resulting in a reduction in SF. Since the velocity of the expelled gas does not exceed the escape velocity (Muratov et al. 2015), it soon cools, re-accretes back to the center, and resumes SF. This periodic gas displacement across the galaxy creates fluctuations in the central gravitational potential, which in turn leads to variation in the stellar distribution and the measured size of the galaxy. This theoretical picture can explain the relationship between the FUV luminosity and size in our observed low-mass sample.

However, it is not yet understood why this dependency is less effective between the size fluctuation and short-timescale SFR indicators, i.e., $\mathrm{H} \alpha$ luminosity. Since $\mathrm{H} \alpha$ and FUV luminosities trace the SFR on different timescales (Flores Velázquez et al. 2021), a detailed investigation on the timescale over which the galaxy size fluctuation and SF variation occur is critical. In this section we aim to compare the SFHs with the half-light radius time sequences for a set of simulated galaxies in order to find quantitative explanations for our observed findings.

Feedback in Realistic Environments (FIRE-1, FIRE-2; Hopkins et al. 2014, 2018) is a set of simulations with models for SF and stellar feedback implemented within cosmological baryonic simulations. In the FIRE model, energy and momentum inputs from stars are taken from stellar evolution calculations and models of the unresolved Sedov-Taylor phase, without tunable parameters. The simulations have been successful in reproducing several key observables, including realistic galactic outflows (Muratov et al. 2015, 2017), the dense HI content of galaxy halos (Faucher-Giguère et al. 2015), the mass-metallicity relation (Ma et al. 2016), the masssize relation and age/metallicity gradients (El-Badry et al. 2016), cored dark matter profiles (Chan et al. 2015; Oñorbe et al. 2015), stellar kinematics (Wheeler et al. 2017), the Kennicutt-Schmidt relation (Orr et al. 2018), observed abundance distributions (Escala et al. 2018), and a realistic population of satellites around Milky Way-mass hosts (Wetzel et al. 2016). 

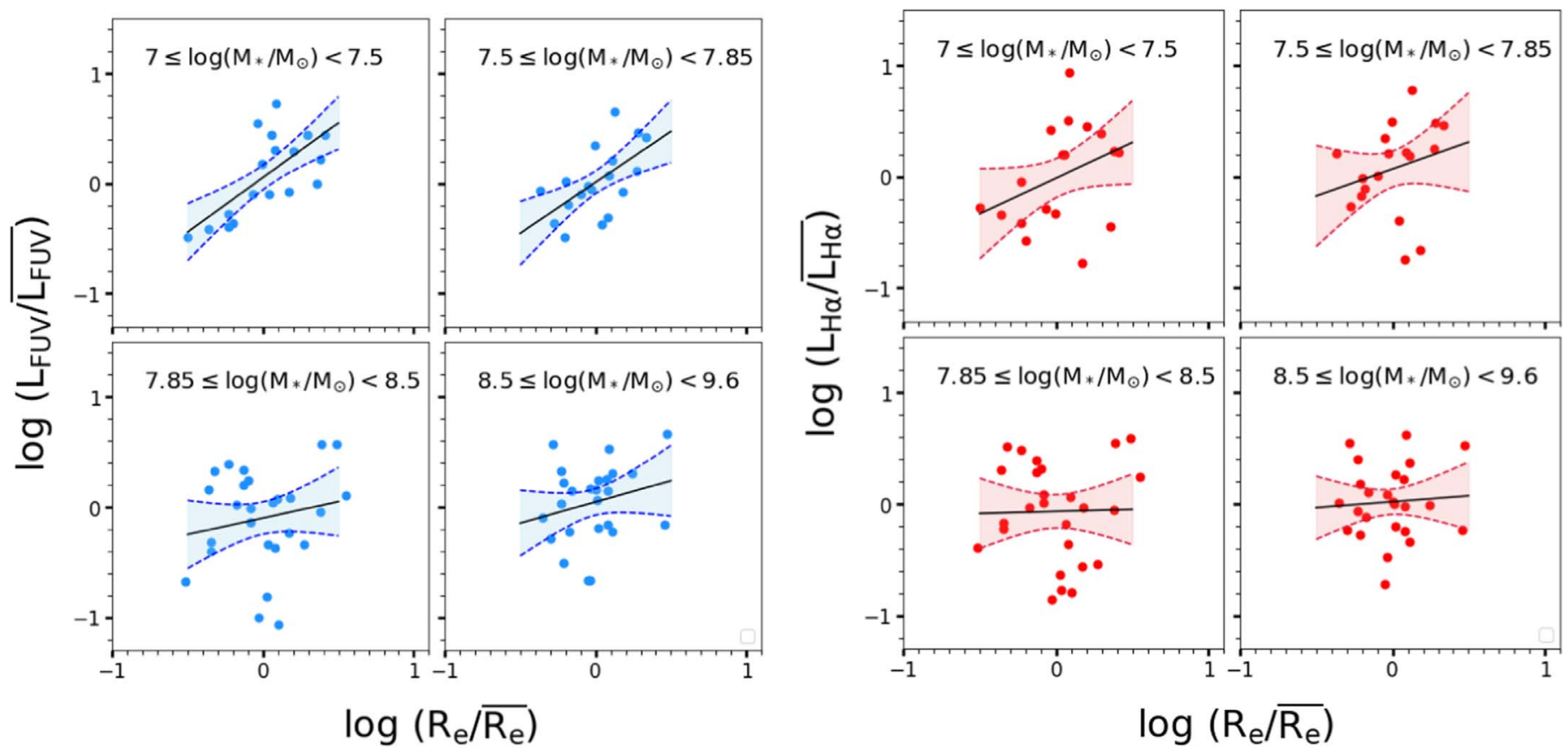

Figure 4. Left: logarithm of normalized-to-average FUV luminosity density $\left(L_{\mathrm{FUV}} / \overline{L_{\mathrm{FUV}}}\right)$ vs. logarithm of normalized-to-average half-light radius $\left(R_{e} / \overline{R_{e}}\right)$. A linear fit and $1 \sigma$ scatter are shown as black lines and blue shaded areas, respectively, for each mass bin. There is a clear correlation at the $10^{7} M_{\odot} \leqslant M_{*}<10^{7.85} M_{\odot}$ mass range, which becomes weaker at larger masses. This can be interpreted as follows: bursty SF causes a size fluctuation in low-mass galaxies, and this size fluctuation reacts to the SF change over a timescale similar to that of the FUV luminosity. Right: logarithm of normalized-to-average $\mathrm{H} \alpha \operatorname{luminosity}\left(L_{\mathrm{H} \alpha} / \overline{L_{\mathrm{H} \alpha}}\right)$ vs. logarithm of $\left(R_{e} / \overline{R_{e}}\right)$. There is a positive slope with large scatter at masses below $10^{7.85} M_{\odot}$ that becomes shallower toward larger masses.

Table 1

The Slopes and Spearman Rank Correlations $(\rho)$ of the Logarithm of FUV and $\mathrm{H} \alpha$ Normalized-to-average Luminosities versus Logarithm of Normalized-toaverage Half-light Radius $\left(R_{e} / \overline{R_{e}}\right)$ for Four Stellar Mass Bins in the LVL Sample

\begin{tabular}{lcc}
\hline \hline $\log \left(M_{*} / M_{\odot}\right)$ & $\begin{array}{c}\log \left(L_{\mathrm{FUV}} / \overline{L_{\mathrm{FUV}}}\right)-\log \left(R_{e} / \overline{R_{e}}\right) \\
\text { slope }(\rho)\end{array}$ & $\begin{array}{c}\log \left(L_{\mathrm{H} \alpha} / \overline{L_{\mathrm{H} \alpha}}\right)-\log \left(R_{e} / \overline{R_{e}}\right) \\
\text { slope }(\rho)\end{array}$ \\
\hline $7-7.5$ & $1.00+/-0.28(0.66)$ & $0.70+/-0.40(0.37)$ \\
$7.5-7.85$ & $0.91+/-0.31(0.65)$ & $0.50+/-0.50(0.37)$ \\
$7.85-8.5$ & $0.26+/-0.32(0.03)$ & $0.03+/-0.33(-0.06)$ \\
$8.5-9.6$ & $0.33+/-0.34(0.24)$ & $0.09+/-0.32(0.05)$ \\
\hline
\end{tabular}

Here we use FIRE-2 simulations of eight isolated low-mass galaxies spanning over a mass range of $10^{7}-10^{9.6} M_{\odot}$, analogous to our local observed sample, and explore their SF and size evolution over the past 2 Gyr $(z \sim 0.15-0)$.

\subsection{Simulated Size-SFR Relation}

To calculate the $R$-band half-light radii of the simulated galaxies, we need to first generate mock $R$-band images of each snapshot in the simulation. The time spacing between consecutive snapshots is $\sim 20 \mathrm{Myr}$. Following procedures in Chan et al. (2018), we generate a table of $R$-band luminosity-tomass ratios of single stellar populations for a range of metallicity and age. For that we run the Flexible Stellar Population Synthesis (Conroy et al. 2009, 2010) model and assume the latest Padova stellar evolutionary tracks (Marigo \& Girardi 2007; Marigo et al. 2008), Kroupa initial mass function (Kroupa 2001), and MILES stellar library. We determine the $R$ band luminosity-to-mass ratio of each simulated star particle by interpolating the generated table at the particle's age and metallicity and then multiply the resulting luminosity-to-mass ratio by the mass of the star particle to get the $R$-band luminosity. We then project the $R$-band luminosities of each simulation output over a $20 \mathrm{kpc} \times 20 \mathrm{kpc}$ region onto $1400^{2}$ mesh grids and generate mock $R$-band images. We have examined our analysis on a larger number of mesh grids and found our size measurements robust to spatial resolutions. Since most of these galaxies are spherical, the size measurements are independent from the orientation we choose to view the galaxy. We show snapshots of $R$-band mock images of galaxy $\mathrm{m} 11 \mathrm{c}$ in Figure 5, which indicate that the galaxy undergoes a significant size fluctuation across $~ 150 \mathrm{Myr}$.

We calculate the half-light radius for each snapshot in all simulated galaxies by passing the $R$-band mock images to Source Extractor and use the same input parameters as those used for our observed galaxies (See Section 2). We also use a point-spread function (PSF) equivalent to the median seeing of our observed sample (1!"4) assuming that the simulated galaxies are at a $5 \mathrm{Mpc}$ distance (which is the median distance of the observed sample) and find that the chosen PSF is much smaller than the galaxy sizes. In order to account for the potential impact of sky noise on our size measurements, we add stochastic sky backgrounds with an average surface brightness $\sim 26$ mag $\operatorname{arcsec}^{-2}$ to our mock images. We find that the differences in the estimated half-light radii with and without sky backgrounds are small and do not affect our analysis. Figure 6 shows the $R$-band half-light radius as a function of stellar mass for FIRE-2 galaxies. The distribution of the simulated galaxies is consistent with that of the observed LVL galaxies in the $R_{e}$-mass parameter space. Also, similar to our observed sample, we find our size measurements to be in agreement with those of $\mathrm{z} \sim 0$ SDSS galaxies (Blanton et al. 2011). We also see that $R_{e}$ fluctuates by more than a factor of 2 over 200 Myr in most of the galaxies. 


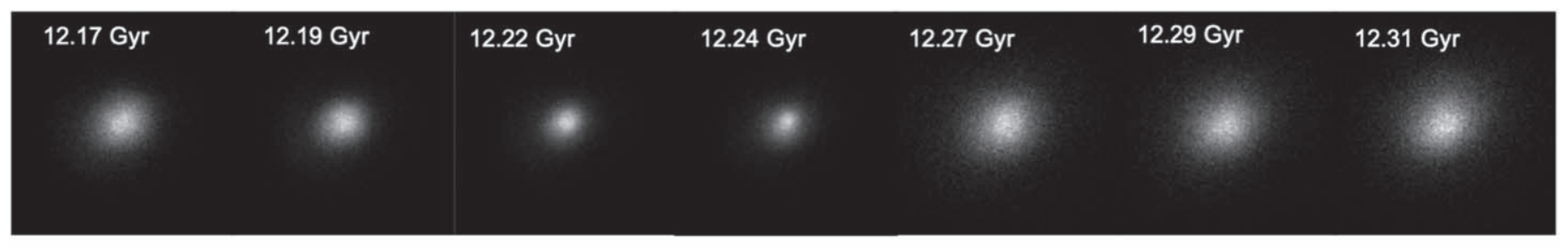

\section{$5 \mathrm{kpc}$}

Figure 5. Snapshots of $R$-band mock images of galaxy m11c in FIRE-2 simulations. It is evident that the simulated galaxy undergoes a significant size fluctuation across $\sim 150$ Myr.

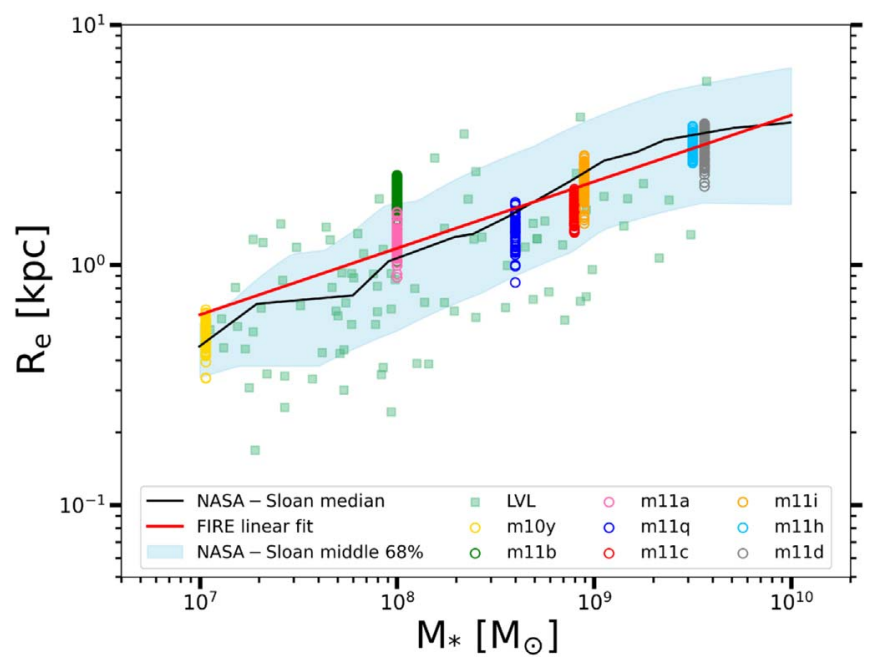

Figure 6. $R$-band half-light radius $\left(R_{e}\right)$ as a function of stellar mass in FIRE-2 galaxies. $R_{e}$ is measured in 100 snapshots during the past 2 Gyr. The red line is the linear fit to the FIRE-2 data points. The sizes measured for the FIRE-2 galaxies are consistent with those of the observed LVL galaxies (green squares). The blue shaded area and the black curve are the median and $1 \sigma$ scatter, respectively, for the NASA-Sloan Atlas at $z \sim 0$ (Blanton et al. 2011). The simulated galaxies are located within the $1 \sigma$ scatter in the NASA-Sloan sample, and the median of the NASA-Sloan sample agrees very well with the FIRE-2 line fit.

\subsection{Size Fluctuation as a Consequence of Bursty Star Formation}

Next, we compare the SFHs with the $R_{e}$ time sequences of the simulated galaxies. Figure 7 shows the SFH and $R_{e}$ time sequences for galaxy $\mathrm{m} 11 \mathrm{q}\left(10^{8.6} M_{\odot}\right)$. The local minima and maxima in the $R_{e}$ time sequences coincide with certain phases in the $\mathrm{SFH} ; R_{e}$ is at its lowest when $\mathrm{SF}$ has just begun rising (nearly after a few Myr; green arrows), and it peaks once SF shuts down (purple arrows). The physical interpretation is that when SF has just begun rising, gas in the center is highly concentrated and forms stars at small radii, and that the majority of the stellar mass is in the center. After supernovae begin to explode, the energetic outflowing winds drive away the gas, resulting in a shallow central gravitational potential that drives stars to outer radii. The subsequent supernovae cumulatively drive gas and stars farther away, and the galaxy expands and reaches its maximum size. Once SF has completely shut off, the central potential returns to a relaxation phase, the cooled gas and stars reaccrete, and the galaxy starts to contract until the next burst cycle begins. We see this phenomenon in all of our simulated galaxies at a mass range of $10^{7}-10^{9.6} M_{\odot}$. Based on this argument and Figure 7 , it can be inferred that the size of the galaxy traces the SF changes with a time delay.

\subsection{What Is the Time Delay from SF Change to Galaxy Size Change?}

In order to determine how long it takes the size of a galaxy to respond to the SF change, i.e., the time delay from SF change to the galaxy size change, we calculate the cross-correlation between the $R_{e}$ time sequence and SFH for each galaxy in the FIRE-2 sample. Since our $R_{e}$ data points are spaced by $20 \mathrm{Myr}$ while our SFRs are sampled at every single Myr, it is very important to first smooth the time sequence of the latter to a time resolution close to that of $R_{e}$ and then sample the time sequences for $20 \mathrm{Myr}$ intervals; otherwise, it is likely that the long-term SF fluctuations will be lost behind the instantaneous SF fluctuations.

In the cross-correlation algorithm, the correlation between $R_{e}$ and SFR time sequences with lag $k$ is defined as

$$
\sum_{n} R_{e}[n+k] . \mathrm{SFR}^{*}[n]
$$

where $\operatorname{SFR}^{*}[n]$ is the complex conjugate of SFR at the $n$th snapshot and $R_{e}[n+k]$ is the $R_{e}$ value at the $(n+k)$ th snapshot. Figure 8 shows the cross-correlation between $R_{e}$ and SFR as a function of time lag for galaxy $\mathrm{m} 11 \mathrm{q}$. The peak in the crosscorrelation plot indicates the time lag between the SFH and $R_{e}$ time sequences, by which the $R_{e}$ time sequences correlate best with the SFH if the SFH is shifted by that amount. We show the same plots for the rest of the sample in Figure 11 in the Appendix. The time lag at the maximum cross-correlation is reported in Table 2. In six out of eight galaxies, the crosscorrelation peaks at $\sim 50 \mathrm{Myr}$ time lag. This implies that it takes the galaxy's size and the stellar migrations roughly $50 \mathrm{Myr}$ to react to any changes in the SFR. For the other two galaxies (m11b, m11h), the cross-correlation peaks at zero Myr time lag between $R_{e}$ and SFR. This is because in these two galaxies the starbursts occur immediately one after another such that there is no quenching time between consecutive bursts, making it difficult for the cross-correlation algorithm to identify individual extrema in the $\mathrm{SFH}$ and $R_{e}$ time sequences.

We also perform the cross-correlation algorithm on three SFR observables with different timescale sensitivities, including the $\mathrm{H} \alpha$ luminosity and FUV (1500 $⿱$ A) and near-UV (NUV; $3500 \AA$ ) luminosity densities. Our goal is to see which one of these SFR observables has the shortest time lag with the $R_{e}$ and thus is better (positively) correlated with the galaxy size. The medians of the time lags between $R_{e}$ and $\mathrm{H} \alpha$, FUV, and NUV for all the galaxies are 42,28 , and $8 \mathrm{Myr}$, respectively. This 


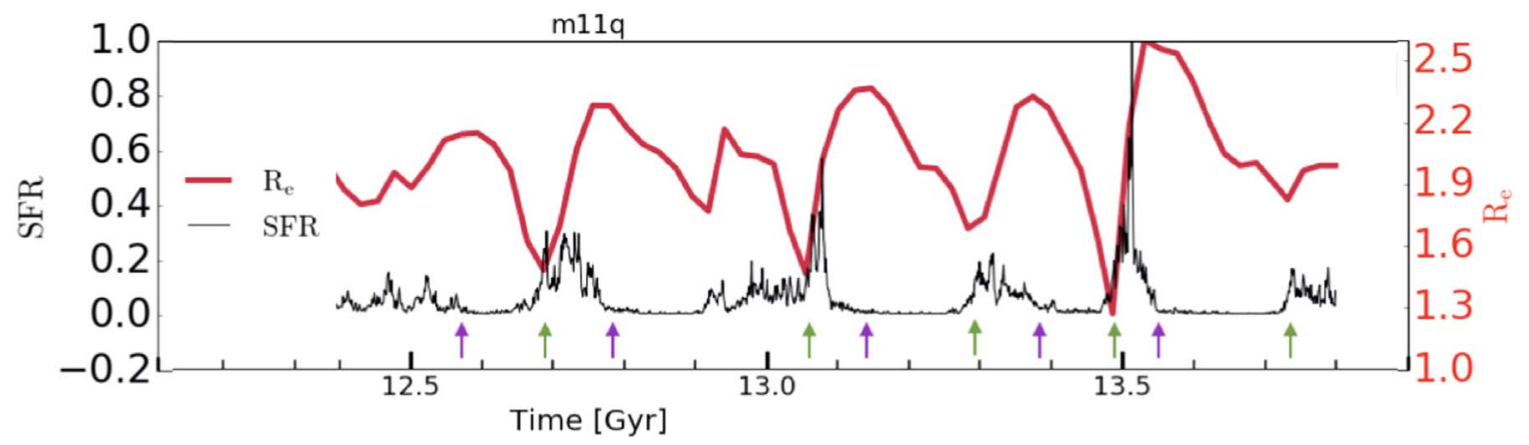

Figure 7. Half-light radius $\left(R_{e}\right)$ time sequence (red) and SFH (black) for galaxy m11q. Starbursts with time durations of 100-200 Myr are evident in the SFH, consistent with the durations of starbursts observed in the local low-mass galaxies (McQuinn et al. 2012). There is a delay in the $R_{e}$ to respond to the SFR variations. $R_{e}$ is at its lowest when SFR has just begun rising (green arrows) and is at its highest once the SFR shuts down (purple arrows). It can be inferred that episodic bursts of SF power energetic outflowing winds that drive away gas, shallow the gravitational potential, and cause the stars to move outward. This causes the galaxy's size to fluctuate dramatically on few $\times 10^{2}$ Myr timescales.

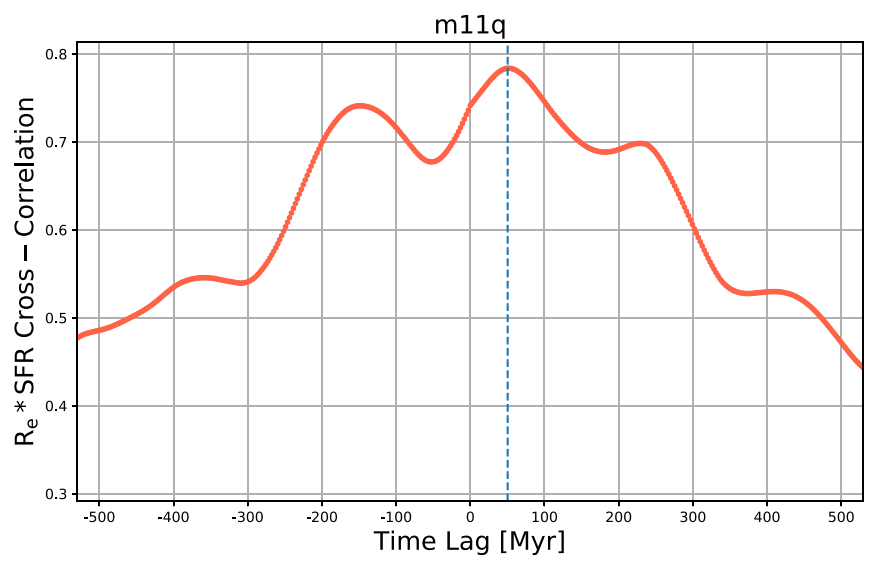

Figure 8. Cross-correlation of half-light radius $\left(R_{e}\right)$ with SFR for galaxy m11q. The peak of the cross-correlation function at time lag $=50 \mathrm{Myr}$ suggests that the galaxy size traces the SF change with a 50 Myr delay. The cross-correlation functions for other FIRE-2 galaxies are provided in the Appendix.

Table 2

Time Lag at the Maximum Cross-correlation between SFR and Half-light Radius $\left(R_{e}\right)$ in FIRE-2 Galaxies

\begin{tabular}{lcc}
\hline \hline Name & $\begin{array}{c}\log \left(M_{*} / M_{\odot}\right) \\
z \sim 0\end{array}$ & $\begin{array}{c}R_{e}-\text { SFR Time Lag (Myr) } \\
\text { (Cross-correlation) }\end{array}$ \\
\hline m10y & 7.03 & 48 \\
m11a & 8.0 & 52 \\
m11b & 8.0 & 0 \\
m11q & 8.6 & 50 \\
m11c & 8.9 & 63 \\
m11i & 8.95 & 43 \\
m11h & 9.5 & 0 \\
m11d & 9.56 & 56 \\
\hline
\end{tabular}

means that $R_{e}$ and NUV luminosity are more correlated with each other (compared to the $R_{e}$ with $\mathrm{H} \alpha$ or FUV) and NUV luminosity traces the SFR on a comparable timescale to the $R_{e}$ changes. This is expected given that the FUV and $\mathrm{H} \alpha$ light comes from short-lived, massive, and hot stars that trace the SF change on a short timescale, while NUV light comes from longer-lived, less massive stars that take a longer time to trace the SF variations.

In Figure 9 we show the normalized $R_{e}$ time sequences along with the normalized SFH that lags by $50 \mathrm{Myr}\left(\mathrm{SFR}_{50}\right)$, as well as the normalized NUV, FUV, and $\mathrm{H} \alpha$ time sequences for

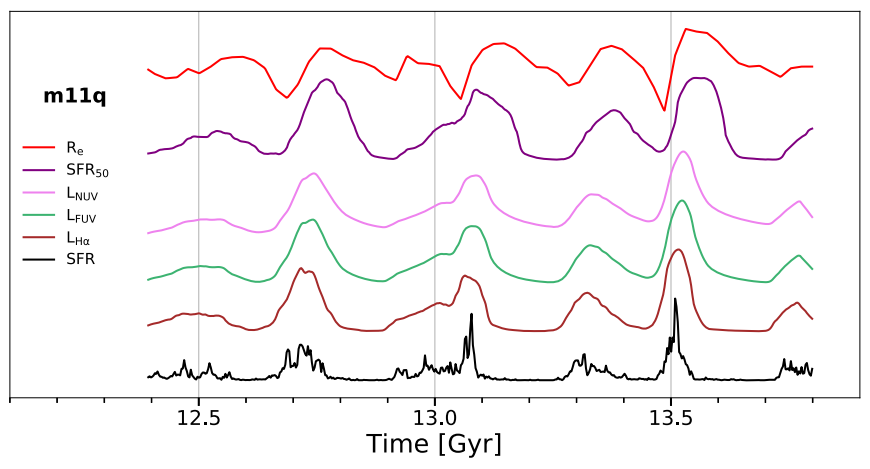

Figure 9. Normalized half-light radius $\left(R_{e}\right)$ time sequences (red) compared with the normalized time sequences of different SFR indicators in galaxy m11q. SFR indicators are sorted based on their correlation with the $R_{e}$ time sequence, with the highest correlation at the top $\left(\mathrm{SFR}_{50}\right.$, the SFR that is lagged by $50 \mathrm{Myr})$ and the lowest correlation at the bottom $\left(L_{\mathrm{H} \alpha}\right)$. Major episodes in $R_{e}$ are very well in phase with the major episodes in the $\mathrm{SFR}_{50}$ time sequence and are completely out of phase with that of $L_{\mathrm{H} \alpha}$. The SFH is also shown in black for reference. The complete figure set is available in the online journal for the rest of the FIRE-2 sample.

(The complete figure set (7 images) is available.)

galaxy m11q. It is evident that an SFH with a $50 \mathrm{Myr}$ time lag is well in phase with the $R_{e}$ time sequence. The correlation between $R_{e}$ and the three SFR observables decreases from NUV, to FUV, to $\mathrm{H} \alpha$ luminosity, respectively, until the $R_{e}$ and $\mathrm{H} \alpha$ time sequences become fully uncorrelated. This can also be seen in almost all of the FIRE-2 galaxies (see online journal for the rest of the FIRE-2 sample).

In order to directly compare these results from simulated galaxies with our observed results in Section 3, we plot all the SFR indicators as a function of $R_{e}$ in Figure 10 and fit a line to each distribution. We present the slopes and the Spearman rank correlation coefficients $(\rho)$ in Table 3 . It is evident that there is a positive slope in the $R_{e}-\mathrm{SFR}_{50}$ relation and the slope decreases from left to right, ultimately reaching an uncorrelated $R_{e}-L_{\mathrm{H} \alpha}$. We also see a shallow FUV $-R_{e}$ correlation in most of the galaxies, which is in agreement with our observed findings in Figure 4. Unlike in the observations, however, we do see a correlation at all masses. This is in tension with what we found in our observed sample, in which the correlation was stronger at masses below $10^{7.85} M_{\odot}$ and became less correlated toward higher masses. This is due to the bursty nature of SF in FIRE simulations, in which the timescale of SF bursts is much shorter than the timescale implied by observations of real galaxies at 

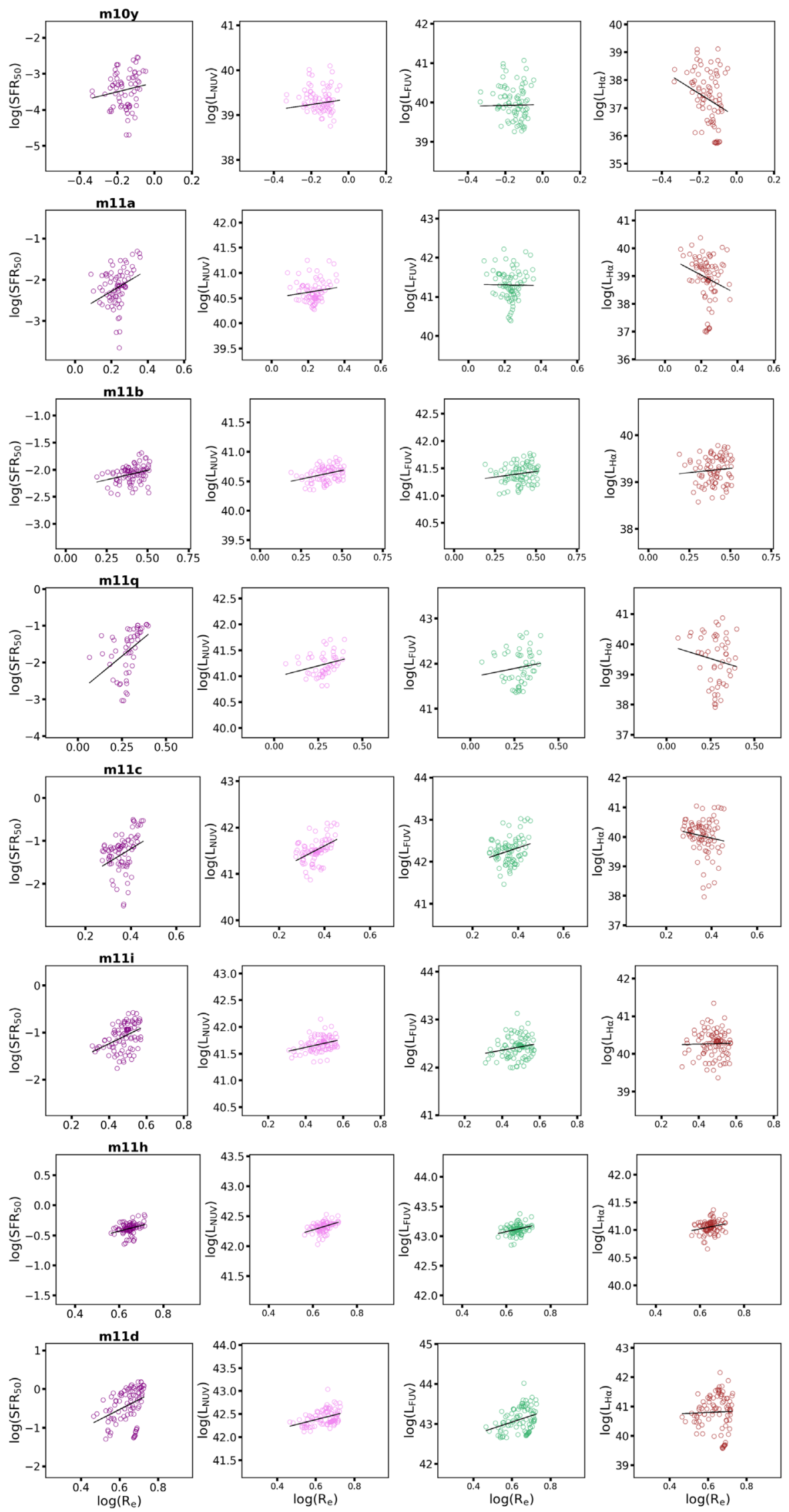

Figure 10. Logarithm of different SFR indicators as a function of logarithm of half-light radius $\left(R_{e}\right)$ in FIRE-2 galaxies. SFR indicators are sorted based on their correlation with $R_{e}$, with the highest correlation at left $\left(\mathrm{SFR}_{50}\right)$ and the lowest correlation at right $\left(L_{\mathrm{H} \alpha}\right)$. The slope decreases from left to right in most galaxies, which is an emphasis on what has been found in Figures 8 and 9, that the $R_{e}$ correlates best with the SFR that is lagged by 50 Myr. 
Table 3

Slopes and the Spearman Rank Correlation $(\rho)$ of the $\log \left(R_{e}\right)-\log (\mathrm{SFR})$ Relation for Different SFR Indicators in Each FIRE-2 Galaxy

\begin{tabular}{|c|c|c|c|c|}
\hline Name & $\begin{array}{l}R_{e}-\mathrm{SFR}_{50} \\
\text { Slope }(\rho)\end{array}$ & $\begin{array}{l}R_{e}-L_{\mathrm{NUV}} \\
\text { Slope }(\rho)\end{array}$ & $\begin{array}{l}R_{e}-L_{\mathrm{FUV}} \\
\text { Slope }(\rho)\end{array}$ & $\begin{array}{c}R_{e}-L_{\mathrm{H} \alpha} \\
\text { Slope }(\rho)\end{array}$ \\
\hline $\mathrm{m} 10 \mathrm{y}$ & $1.28 \pm 1(0.03)$ & $0.62 \pm 1.38(-0.08)$ & $0.12 \pm 1.56(-0.11)$ & $-4.22 \pm 2.25(-0.30)$ \\
\hline $\mathrm{m} 11 \mathrm{a}$ & $2.61 \pm 1.05(0.32)$ & $0.59 \pm 0.47(0.12)$ & $-0.07 \pm 0.86(-0.04)$ & $-3.47 \pm 1.73(-0.28)$ \\
\hline $\mathrm{m} 11 \mathrm{q}$ & $3.94 \pm 1.16(0.67)$ & $0.88 \pm 0.44(0.38)$ & $0.78 \pm 0.80(0.24)$ & $-1.80 \pm 1.73(-0.08)$ \\
\hline $\mathrm{m} 11 \mathrm{c}$ & $3.20 \pm 1.22(0.39)$ & $2.53 \pm 0.55(0.48)$ & $1.80 \pm 0.76(0.17)$ & $-1.79 \pm 1.51(-0.16)$ \\
\hline $\mathrm{m} 11 \mathrm{i}$ & $1.93 \pm 0.44(0.44)$ & $0.77 \pm 0.23(0.33)$ & $0.72 \pm 0.38(0.16)$ & $0.11 \pm 0.61(-0.04)$ \\
\hline
\end{tabular}

high mass (Muratov et al. 2015; Sparre et al. 2017; Emami et al. 2019; Stern et al. 2021). Furthermore, we see that in Figure 10 , for all four SFR indicators $\left(\mathrm{SFR}_{50}, L_{\mathrm{NUV}}, L_{\mathrm{FUV}}\right.$, and $L_{\mathrm{H} \alpha}$ ), the slopes are biased by a few points that show very low SFR. These points are associated with the post-burst phases that are followed by long periods of no SF. In these cases, SFR decreases rapidly, while $R_{e}$ takes a longer time to decrease and reach an equilibrium. One example can be seen in Figure 9, for which during the quenching time at $13.55 \mathrm{Gyr}$ and the subsequent burst at 13.75 Gyr SFR decreases rapidly while $R_{e}$ decreases at a much lower rate.

\section{Discussion}

\subsection{Previous studies}

Patel et al. (2018) have tested the effect of bursty SF on the galaxy size fluctuation for a sample of intermediate-mass galaxies (IMGs; $10^{9}-10^{9.5} M_{\odot}$ ) at a redshift range of $0.3-0.4$ in the Hubble Space Telescope $I_{814}$ Cosmological Evolution Survey footprint. They measured the SFRs based on combined $\mathrm{UV}$ and IR luminosities and looked at the $R_{e}-\mathrm{SSFR}$ relation in their sample. There are some differences between their analyses and ours. The key difference is that Patel et al. (2018) did not subtract off the mass dependency from their $R_{e}$ measurements. Thus, it becomes impossible to infer whether a large $R_{e}$ is because of an expansion due to burstiness or because of an intrinsically massive large galaxy. Despite that, they found that IMGs with higher sSFRs are the most extended, with median sizes of $R_{e} \sim 2.8-3.4 \mathrm{kpc}$, and IMGs with lower sSFRs are a factor of $\sim 2-3$ more compact, with median sizes of $R_{e} \sim 0.9-1.3 \mathrm{kpc}$. This is consistent with our observed $\log$ $\left(R_{e} / \overline{R_{e}}\right)-\log \left(\mathrm{sSFR}_{\mathrm{FUV}}\right)$ correlation for our $10^{7}-10^{7.85} M_{\odot}$ local galaxies. They concluded that their observed $R_{e}-\mathrm{SSFR}$ correlation is in tension with what simulations predict, i.e., the size should decrease when SF is in a burst and increase when $\mathrm{SF}$ is quenched. However, we find that both the FUV luminosity and the size of the galaxy trace SF change on different timescales, and the UV luminosity should not be mistaken for the instantaneous SFR.

As we discussed earlier in Section 1, bursty SF is predicted to drive fluctuations for both gas and star particles in low-mass galaxies in the form of gas emission line broadening and galaxy size fluctuations, respectively (El-Badry et al. 2016). In this paper, we have tested the effect of burstiness on the galaxy size fluctuations and found that the size of the galaxy is coupled with SF that is delayed by $50 \mathrm{Myr}$. Furthermore, there have been observational studies on the SFHs and its relationship with neutral hydrogen ( $\mathrm{H} \mathrm{I}$ ) gas velocities of a sample of local dwarf galaxies (Stilp et al. 2013). Stilp et al. (2013) found that $\mathrm{H}$ I line broadening, or equivalently $\mathrm{H} \mathrm{I}$ energy surface density, is strongly correlated only with SF that occurred 30-40 Myr ago. They argue that the coupling between SF and the neutral interstellar medium is strongest on this timescale, due either to an intrinsic delay between the release of the peak energy from SF or to the coherent effects of many supernova explosions during this interval. The timescale found by Stilp et al. (2013) is very similar to the one found in this work ( $\sim 50 \mathrm{Myr})$, and these two findings together support the theoretical predictions that bursty SF impacts both stellar and gas kinematics in lowmass systems.

\subsection{The Role of Galaxy Formation Histories and Mergers in the $R_{e} / \overline{R_{e}}-S F R$ Relation}

We caution that the fact that bursty SF can drive size fluctuations does not mean that the size fluctuations are solely driven by burstiness, and in reality there might be other factors that might contribute to the size fluctuations at each stellar mass.

We further tested to see whether the relationship between the mass-subtracted sizes $\left(R_{e} / \overline{R_{e}}\right)$ and the SFRs seen in Figure 4 are caused by other factors than the burstiness, for instance, the galaxy formation histories or the galaxy-galaxy interactions. Galaxies that have formed earlier indicate small $R_{e}$ and tend to lack late-time SFs, which reflects as low SFRs in both $\mathrm{H} \alpha$ - and UV-derived SFRs. Therefore, there might be a number of earlytype galaxies that contribute to our observed $R_{e} / \overline{R_{e}}-\mathrm{SFR}$ trend in Figure 4. Since the $\mathrm{H} \alpha$ and UV luminosities of these galaxies are equally low, their $L_{\mathrm{H} \alpha} / L_{\mathrm{UV}}$ approaches the equilibrium value of $10^{-2.12}$ (Emami et al. 2019). However, a plot of $\log \left(L_{\mathrm{H} \alpha} / L_{\mathrm{UV}}\right)$ of the LVL sample (Emami et al. 2019, Figure 4) shows that the low mass bins, i.e., masses below $10^{8}$ $M_{\odot}$, span a large range of $L_{\mathrm{H} \alpha} / L_{\mathrm{UV}}$. This implies that galaxies in our LVL sample undergo recent SFs and the $R_{e} / \overline{R_{e}}-\mathrm{SFR}$ trend that we see in our observed sample cannot be driven by the early-type dwarfs. Furthermore, galaxies that are undergoing merger processes indicate an increase in their SFRs and an increase in their half-light radii due to their tidal tails. Therefore, interacting systems can also result in a rising $R_{e} / \overline{R_{e}}-$ SFR trend. However, we found that none of our galaxies exist in the catalog of known merging local dwarf galaxies (Paudel et al. 2018). Hence, it is very unlikely that the galaxy formation histories or mergers contribute to the $R_{e} / \overline{R_{e}}-$ SFR trend that we see in our observed sample. 


\subsection{Implications for High-redshift Tests with the James Webb Space Telescope}

Our observed findings, combined with the findings from the FIRE-2 simulations, suggest that the size of a galaxy traces the bursty SF with a delay of approximately 50 Myr. Given that bursty SF is more prominent at high redshifts (Guo et al. 2016; Caplar \& Tacchella 2019; Faisst et al. 2019), it is important that we study the effect of burstiness on size fluctuations in a large and complete dwarf galaxy sample at high redshifts. However, at high redshifts, the photometric aperture in the rest-frame UV and observed optical bands is severely biased toward compact regions of galaxies where the undergoing bursts occur, and the more extended, old underlying regions are often unresolved (Zick et al. 2018; Ma et al. 2018; see also Zick et al. 2021, in preparation). This is due to the fact that the extended underlying regions fall below the surface brightness threshold of the current instruments (e.g., Hubble Space Telescope), leading us to underestimate the true size of the galaxies at high redshifts. With the advent of the James Webb Space Telescope and its high surface brightness sensitivity in the near-IR imaging (NIRCam), we can detect the lower surface brightness stellar populations in the galaxies and investigate the morphological effects of bursty SF on galaxies at early epochs.

\section{Summary}

We explored the effect of bursty SF on galaxy sizes, which results from the implementation of stellar feedback onto the dark-matter-only simulations. Winds driven by outflows induce fluctuations on the gravitational potential and cause a radial stellar migration in low-mass galaxies. We tested this prediction on samples of both observed local dwarf galaxies and simulated late-time dwarfs across a mass range of $10^{7}-10^{9.6} M_{\odot}$, where theoretical simulations suggest feedback to be most efficiently altering galaxies' kinematics (El-Badry et al. 2016). For our observed sample, we used $\mathrm{H} \alpha$ and FUV luminosities ( $\left.L_{\mathrm{FUV}}\right)$ to probe different timescales of the SFR and the $R$-band images to measure the half-light radii $\left(R_{e}\right)$. We found that the half-light radius correlates well with $L_{\mathrm{FUV}}$ while the correlation becomes weaker between $R_{e}$ and $L_{\mathrm{H} \alpha}$. Furthermore, the $R_{e}-L_{\mathrm{FUV}}$ relation is much more significant at masses below $\sim 10^{8} M_{\odot}$ and becomes shallower at higher masses. These findings suggest that the half-light radius changes owing to the SF variations and reacts to this variation within a timescale similar to that of FUV luminosity (i.e., $10 \mathrm{Myr}$ or longer), but not close to the $\mathrm{H} \alpha$ timescale at all, which is a few Myr. To understand the underlying physics of this size-SFR correlation and determine the approximate timescale over which the size traces the SFR variations, we explored eight low-mass galaxies from the FIRE2 cosmological simulations at late cosmic times (over the past 2 Gyr) that mimic our observed local sample. We summarize our findings as follows:

1. By visual inspections of the half-light radius time sequence and the SFH of galaxies, it is evident that there is a delay from the onset of a burst of SF to when halflight radius increases. The physical interpretation is that when SF begins rising, gas and stars are highly concentrated and the galaxy is compact. After subsequent supernovae, the resulting outflows drive gas and stars to larger radii and the galaxy expands. This expansion continues until the SF completely shuts down and then cooled gas and stars migrate back to the center.

2. In order to assess the timescale by which $R_{e}$ traces the SF change, we performed the cross-correlation technique. We found that there is a 50 Myr time lag between the SFH and half-light radius in almost all galaxies, suggesting that the size of the galaxy traces the SF changes with a 50 Myr delay. This is also evident as a positive slope in the half-light radius-SFR ${ }_{50}$ relation for almost all the FIRE galaxies.

3. Using multiple common SFR observables, including the $\mathrm{H} \alpha$, FUV, and NUV luminosities, and cross-correlating these with the half-light radius, we found that the time sequence of NUV luminosity is more correlated with the half-light radius time sequence, and the correlation decreases for the FUV and then $\mathrm{H} \alpha$ time sequences, respectively. This is consistent with our observed results, for which half-light radius is better correlated with the FUV luminosity than the $\mathrm{H} \alpha$ luminosity.

4. The half-light radius-SFR correlation exists in all the FIRE galaxies spanning a mass range of $10^{7}-10^{9.6} M_{\odot}$. This is in tension with what we found in our observed sample, in which the correlation can only be seen in masses below $10^{7.85} M_{\odot}$. This is likely related to the bursty nature of most massive galaxies in the FIRE simulations at low redshifts (Sparre et al. 2017; Emami et al. 2019).

5. With the high surface brightness sensitivity of the near-IR imaging cameras on the James Webb Space Telescope, we will be able to test the effect of bursty SF on the size of the high-redshift galaxies, while the biases against low surface brightness galaxies are significantly minimized.

We thank the anonymous referee for providing constructive comments that helped improve the quality of this paper. We also thank T. K. Chan and Amanda Pagul for their helpful discussions. This material is based on work supported by UC Riverside Overhead Return Grant under grant No. A0186819900-40-FPBS.

\section{Appendix}

Figure 11 shows the cross-correlation between Re and SFR as a function of time lag for FIRE-2 galaxies same as the plot shown in Figure 8 for galaxy m11q. The peak in the crosscorrelation plots indicate the time lag between the SFH and Re time sequences, by which the Re time sequences correlate best with the SFH if the SFH is shifted by that amount. The time lag at the maximum cross-correlation is reported in Table 2. In six out of eight galaxies, the crosscorrelation peaks at $\sim 50 \mathrm{Myr}$ time lag. This implies that it takes the galaxy's size and the stellar migrations roughly $50 \mathrm{Myr}$ to react to any changes in the SFR. 

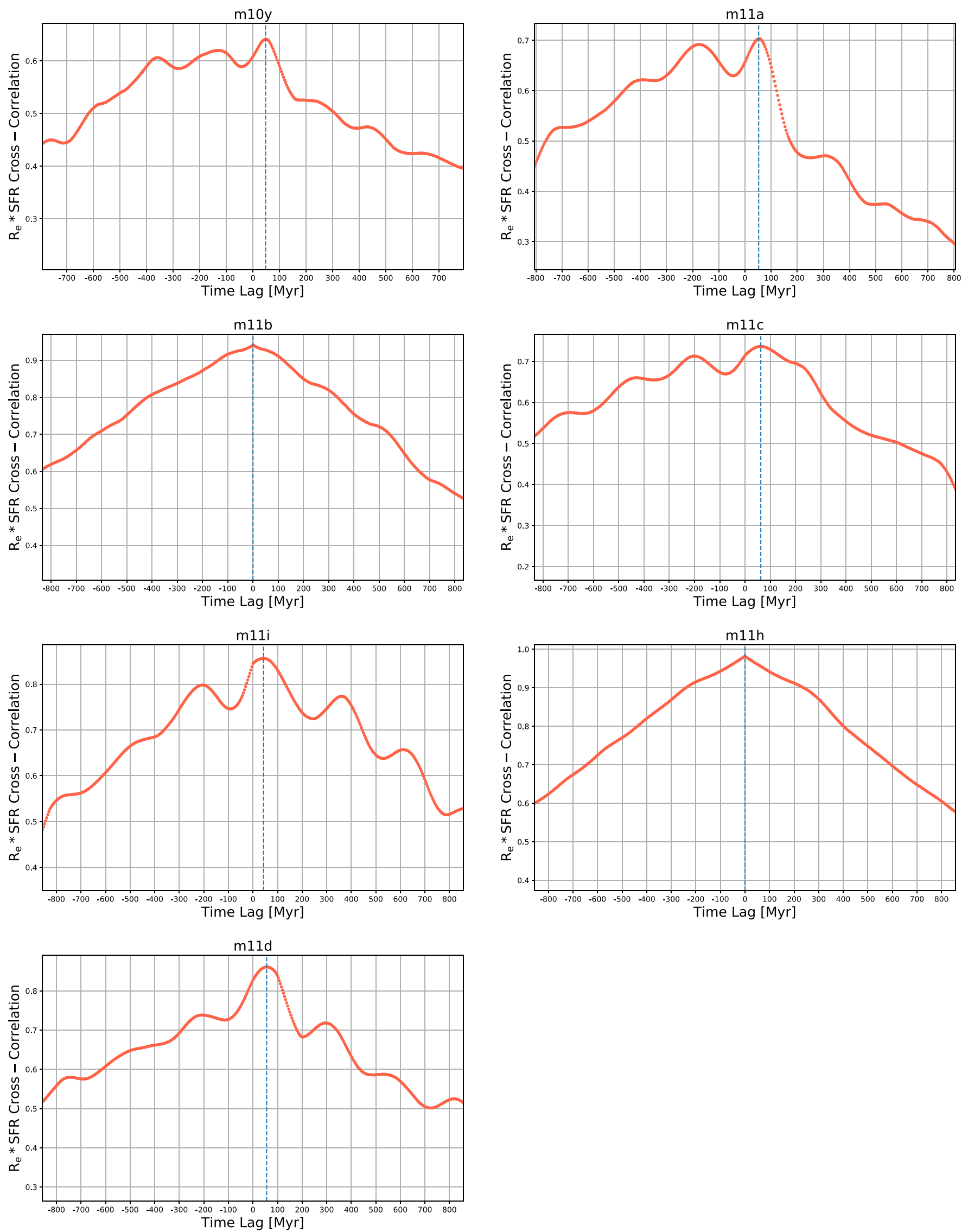

Figure 11. Same as Figure 8, but for the rest of the FIRE-2 galaxies. 


\section{ORCID iDs}

Najmeh Emami (iD https://orcid.org/0000-0003-2047-1689

Brian Siana (i) https://orcid.org/0000-0002-4935-9511

Kareem El-Badry (ib https://orcid.org/0000-0002-6871-1752

David Cook (iD https://orcid.org/0000-0002-6877-7655

Xiangcheng Ma (i) https://orcid.org/0000-0001-8091-2349

Daniel Weisz (iD https://orcid.org/0000-0002-6442-6030

Claudia Scarlata (ib https://orcid.org/0000-0002-9136-8876

Evan Skillman (ib https://orcid.org/0000-0003-0605-8732

\section{References}

Abazajian, K. N., Adelman-McCarthy, J. K., Agüeros, M. A., et al. 2009, ApJS, 182, 543

Aparicio, A., \& Tikhonov, N. 2000, AJ, 119, 2183

Bahcall, N. A., Ostriker, J. P., Perlmutter, S., \& Steinhardt, P. J. 1999, Sci, 284, 1481

Behroozi, P. S., Wechsler, R. H., \& Conroy, C. 2013, ApJ, 770, 57

Berg, D. A., Skillman, E. D., Marble, A. R., et al. 2012, ApJ, 754, 98

Bertin, E., \& Arnouts, S. 1996, A\&AS, 117, 393

Blanton, M. R., Kazin, E., Muna, D., Weaver, B. A., \& Price-Whelan, A. 2011, AJ, 142, 31

Brooks, A. M. 2019, Illuminating Dark Matter, Astrophysics and Space Science Proceedings, Vol. 56 (Berlin: Springer), 19

Bruzual, G., \& Charlot, S. 2003, MNRAS, 344, 1000

Bullock, J. S., \& Boylan-Kolchin, M. 2017, ARA\&A, 55, 343

Caplar, N., \& Tacchella, S. 2019, MNRAS, 487, 3845

Chan, T. K., Kereš, D., Oñorbe, J., et al. 2015, MNRAS, 454, 2981

Chan, T. K., Kereš, D., Wetzel, A., et al. 2018, MNRAS, 478, 906

Cicone, C., Maiolino, R., \& Marconi, A. 2016, A\&A, 588, A41

Conroy, C., Gunn, J. E., \& White, M. 2009, ApJ, 699, 486

Conroy, C., White, M., \& Gunn, J. E. 2010, ApJ, 708, 58

Cook, D. O., Dale, D. A., Johnson, B. D., et al. 2014, MNRAS, 445, 881

Dale, D. A., Cohen, S. A., Johnson, L. C., et al. 2009, ApJ, 703, 517

De Young, D. S., \& Heckman, T. M. 1994, ApJ, 431, 598

Domínguez, A., Siana, B., Brooks, A. M., et al. 2015, MNRAS, 451, 839

El-Badry, K., Wetzel, A., Geha, M., et al. 2016, ApJ, 820, 131

El-Badry, K., Wetzel, A. R., Geha, M., et al. 2017, ApJ, 835, 193

Elbert, O. D., Bullock, J. S., Garrison-Kimmel, S., et al. 2015, MNRAS, 453, 29

Emami, N., Siana, B., Weisz, D. R., et al. 2019, ApJ, 881, 71

Escala, I., Wetzel, A., Kirby, E. N., et al. 2018, MNRAS, 474, 2194

Faisst, A. L., Capak, P. L., Emami, N., Tacchella, S., \& Larson, K. L. 2019 , ApJ, 884, 133

Faucher-Giguère, C.-A., Hopkins, P. F., Kereš, D., et al. 2015, MNRAS, 449, 987

Flores, R. A., \& Primack, J. R. 1994, ApJL, 427, L1

Flores Velázquez, J. A., Gurvich, A. B., Faucher-Giguère, C.-A., et al. 2021, MNRAS, 501, 4812

Frenk, C. S., \& White, S. D. M. 2012, AnP, 524, 507

Fry, A. B., Governato, F., Pontzen, A., et al. 2015, MNRAS, 452, 1468

Governato, F., Brook, C., Mayer, L., et al. 2010, Natur, 463, 203

Graus, A. S., Bullock, J. S., Fitts, A., et al. 2019, MNRAS, 490, 1186

Guo, Y., Rafelski, M., Faber, S. M., et al. 2016, ApJ, 833, 37

Hao, C.-N., Kennicutt, R. C., Johnson, B. D., et al. 2011, ApJ, 741, 124

Hidalgo, S. L., Monelli, M., Aparicio, A., et al. 2013, ApJ, 778, 103

Hirschmann, M., Naab, T., Somerville, R. S., Burkert, A., \& Oser, L. 2012, MNRAS, 419, 3200

Hirtenstein, J., Jones, T., Wang, X., et al. 2019, ApJ, 880, 54

Hopkins, P. F., Grudić, M. Y., Wetzel, A., et al. 2020, MNRAS, 491, 3702

Hopkins, P. F., Kereš, D., Oñorbe, J., et al. 2014, MNRAS, 445, 581

Hopkins, P. F., Wetzel, A., Kereš, D., et al. 2018, MNRAS, 480, 800

Jafariyazani, M., Mobasher, B., Hemmati, S., et al. 2019, ApJ, 887, 204

Johnson, B. D., Weisz, D. R., Dalcanton, J. J., et al. 2013, ApJ, 772, 8

Kaplinghat, M., Tulin, S., \& Yu, H.-B. 2016, PhRvL, 116, 041302
Kennicutt, R. C. J. 1998, ARA\&A, 36, 189

Kennicutt, R. C. J., Hao, C.-N., Calzetti, D., et al. 2009, ApJ, 703, 1672

Kennicutt, R. C. J., Lee, J. C., Funes, J. G., et al. 2008, ApJS, 178, 247

Kron, R. G. 1980, ApJS, 43, 305

Kroupa, P. 2001, MNRAS, 322, 231

Kuzio de Naray, R., McGaugh, S. S., \& de Blok, W. J. G. 2008, ApJ, 676, 920

Lee, J. C., Gil de Paz, A., Kennicutt, R. C. J., et al. 2011, ApJS, 192, 6

Lee, J. C., Gil de Paz, A., Tremonti, C., et al. 2009, ApJ, 706, 599

Ma, X., Hopkins, P. F., Boylan-Kolchin, M., et al. 2018, MNRAS, 477, 219

Ma, X., Hopkins, P. F., Faucher-Giguère, C.-A., et al. 2016, MNRAS, 456, 2140

Marchesini, D., D’Onghia, E., Chincarini, G., et al. 2002, ApJ, 575, 801

Marigo, P., \& Girardi, L. 2007, A\&A, 469, 239

Marigo, P., Girardi, L., Bressan, A., et al. 2008, A\&A, 482, 883

McGaugh, S. S., Rubin, V. C., \& de Blok, W. J. G. 2001, AJ, 122, 2381

McQuinn, K. B. W., Boyer, M. L., Mitchell, M. B., et al. 2017, ApJ, 834, 78

McQuinn, K. B. W., Skillman, E. D., Cannon, J. M., et al. 2010, ApJ, 724, 49

McQuinn, K. B. W., Skillman, E. D., Dalcanton, J. J., et al. 2012, ApJ, 759, 77

Mehta, V., Scarlata, C., Rafelski, M., et al. 2017, ApJ, 838, 29

Mercado, F. J., Bullock, J. S., Boylan-Kolchin, M., et al. 2021, MNRAS, 501,5121

Moore, B. 1994, Natur, 370, 629

Moster, B. P., Naab, T., \& White, S. D. M. 2013, MNRAS, 428, 3121

Muratov, A. L., Kereš, D., Faucher-Giguère, C.-A., et al. 2015, MNRAS, 454, 2691

Muratov, A. L., Kereš, D., Faucher-Giguère, C.-A., et al. 2017, MNRAS, 468,4170

Navarro, J. F., Eke, V. R., \& Frenk, C. S. 1996, MNRAS, 283, L72

Navarro, J. F., Frenk, C. S., \& White, S. D. M. 1997, ApJ, 490, 493

Navarro, J. F., Ludlow, A., Springel, V., et al. 2010, MNRAS, 402, 21

Oh, S.-H., Hunter, D. A., Brinks, E., et al. 2015, AJ, 149, 180

Oñorbe, J., Boylan-Kolchin, M., Bullock, J. S., et al. 2015, MNRAS, 454, 2092

Orr, M. E., Hayward, C. C., Hopkins, P. F., et al. 2018, MNRAS, 478, 3653

Patel, S. G., Kelson, D. D., Diao, N., Tonnesen, S., \& Abramson, L. E. 2018, ApJL, 866, L21

Paudel, S., Smith, R., Yoon, S. J., Calderón-Castillo, P., \& Duc, P.-A. 2018, ApJS, 237, 36

Pelliccia, D., Mobasher, B., Darvish, B., et al. 2020, ApJL, 896, L26

Peng, C. Y., Ho, L. C., Impey, C. D., \& Rix, H.-W. 2002, AJ, 124, 266

Peter, A. H. G., Rocha, M., Bullock, J. S., \& Kaplinghat, M. 2013, MNRAS, 430, 105

Planck Collaboration, Aghanim, N., Akrami, Y., et al. 2020, A\&A, 641, A6

Pontzen, A., \& Governato, F. 2012, MNRAS, 421, 3464

Read, J. I., Iorio, G., Agertz, O., \& Fraternali, F. 2016, MNRAS, 462, 3628

Read, J. I., Walker, M. G., \& Steger, P. 2019, MNRAS, 484, 1401

Relatores, N. C., Newman, A. B., Simon, J. D., et al. 2019, ApJ, 887, 94

Sales, L. V., Marinacci, F., Springel, V., \& Petkova, M. 2014, MNRAS, 439, 2990

Sales, L. V., Navarro, J. F., Schaye, J., et al. 2010, MNRAS, 409, 1541

Sameie, O., Yu, H.-B., Sales, L. V., Vogelsberger, M., \& Zavala, J. 2020, PhRvL, 124, 141102

Schaye, J., Crain, R. A., Bower, R. G., et al. 2015, MNRAS, 446, 521

Simon, J. D., Bolatto, A. D., Leroy, A., Blitz, L., \& Gates, E. L. 2005, ApJ, 621,757

Sparre, M., Hayward, C. C., Feldmann, R., et al. 2017, MNRAS, 466, 88 Spergel, D. N., \& Steinhardt, P. J. 2000, PhRvL, 84, 3760

Stern, J., Faucher-Giguère, C.-A., Fielding, D., et al. 2021, ApJ, 911, 88

Stilp, A. M., Dalcanton, J. J., Warren, S. R., et al. 2013, ApJ, 772, 124

Stinson, G. S., Dalcanton, J. J., Quinn, T., et al. 2009, MNRAS, 395, 1455

Tollet, E., Macciò, A. V., Dutton, A. A., et al. 2016, MNRAS, 456, 3542

Vargas, L. C., Geha, M. C., \& Tollerud, E. J. 2014, ApJ, 790, 73

Vogelsberger, M., Genel, S., Springel, V., et al. 2014, MNRAS, 444, 1518

Vogelsberger, M., Zavala, J., \& Loeb, A. 2012, MNRAS, 423, 3740

Weisz, D. R., Johnson, B. D., Johnson, L. C., et al. 2012, ApJ, 744, 44

Wetzel, A. R., Hopkins, P. F., Kim, J.-h., et al. 2016, ApJL, 827, L23

Wheeler, C., Pace, A. B., Bullock, J. S., et al. 2017, MNRAS, 465, 2420

Zick, T. O., Weisz, D. R., \& Boylan-Kolchin, M. 2018, MNRAS, 477, 480 